
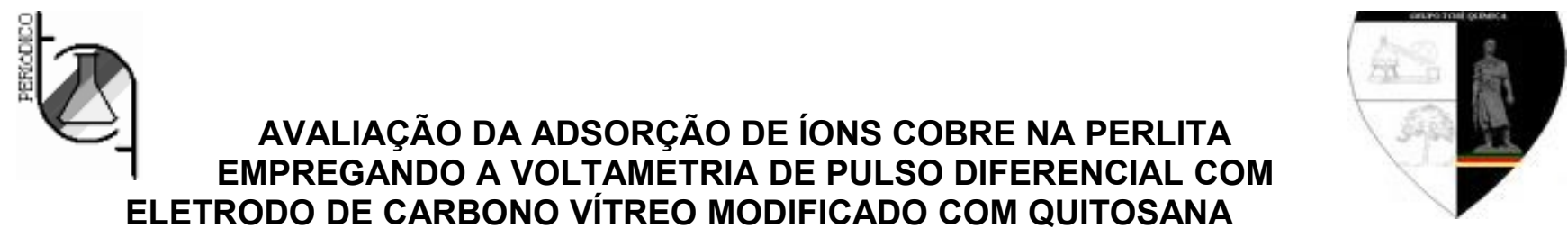

\title{
EVALUATION OF ADSORPTION OF COPPER IONS IN THE PERLITE USING DIFFERENTIAL PULSE VOLTAMMETRY EMPLOYING A CHITOSAN MODIFIED GLASSY CARBON ELECTRODE
}

\author{
NOGUEIRA, James Pyetro do Amaral ${ }^{1}$; CARVALHO, Araújo Daniel ${ }^{1}$, MARTINEZ-HUITLE, Carlos \\ Alberto ${ }^{2}$; FERNANDES, Nedja Suely² \\ 1,2 Universidade Federal do Rio Grande do Norte/UFRN - Departamento de Química Analítica \\ Av. Senador Salgado Filho s/n - Campus Universitário - Lagoa Nova, CEP 59078-970 \\ Natal-RN, Brasil \\ e-mail: nedja@ufrnet.br
}

Received 09 September 2010; received in revised form 06 January 2011; accepted 11 January 2011

\begin{abstract}
RESUMO
Neste trabalho a perlita, um aluminossilicato constituído por $72,1 \%$ de $\mathrm{SiO}_{2}$ e $18,5 \%$ de $\mathrm{Al}_{2} \mathrm{O}_{3}$ foi avaliada como adsorvente de íons cobre em solução aquosa utilizando a voltametria de pulso diferencial a partir da utilização de um eletrodo de carbono vítreo modificado com quitosana (EMQ). Os testes de adsorção realizados no intervalo de 5 a 30 minutos indicaram que $73 \%$ dos íons cobre foram adsorvidos em até 30 minutos.
\end{abstract}

Palavras-chave: perlita, eletrodo de carbono vítreo, quitosana, metais pesados

\begin{abstract}
In this work the perlite, an aluminosilicate constituted by $72.1 \%$ of $\mathrm{SiO}_{2}$ and $18.5 \%$ of $\mathrm{Al}_{2} \mathrm{O}_{3}$ was evaluated as an adsorbent of copper ions in aqueous solution using the differential pulse voltammetry technique employing a chitosan modified glassy carbon electrode (EMQ). The adsorption tests performed in the range from 5 to 30 min indicated that an adsorption of copper ions around $73 \%$ at time of 30 min was achivied.
\end{abstract}

Keywords: perlite; glassy carbon electrode, chitosan, heavy metals 


\section{Introdução}

O descarte por afluentes industriais contaminados por metais pesados vem reduzindo a pureza de rios e solos no meio ambiente. Esses metais, em sua maioria, possuem uma elevada ação tóxica, gerando diversos problemas ambientais, afetando de maneira direta a saúde do homem. O aparecimento de problemas ambientais ligados aos recursos hídricos forçou as autoridades a elaborar leis, programas, políticas públicas e planos para a conservação dos recursos naturais. Aliado a uma constituição de leis ambientais rígidas a respeito dos descartes desses afluentes industriais, tem sido desenvolvidas novas tecnologias com a finalidade de diminuir ou eliminar traços de metais como o cobre, zinco, manganês, cádmio, chumbo, mercúrio, entre outros.

Dentre esses metais, o cobre é um dos poucos que pode ser encontrado em forma nativa na natureza. Devido à sua propriedade de ductilidade, maleabilidade e resistência à corrosão, este vem sendo aplicado na área da construção civil e indústria automobilística, sendo os equipamentos elétricos responsáveis pelo consumo de mais de $60 \%$ deste metal utilizado na indústria. Nesta classe, devem ser incluídos transformadores, equipamentos eletrônicos, transmissão de energia e geradores (HOMEM, 2001).

Concentrações anormais de íons cobre no organismo humano podem ser atribuídas a distúrbios metabólicos, patológicos e absorção excessiva. A deficiência ou acúmulo de cobre na dieta alimentar tem gerado mal funcionamento dos órgãos humanos. As crianças são os mais afetados a respeito dos efeitos da desproporção da concentração desse metal de transição $d$, causando síndromes clínicas como anemia, diarréia e hipocupremia. (FAQUIM,1994)

O efeito biológico causado pelo cobre no meio ambiente tem despertado grande preocupação, uma vez que, dependendo da concentração, este apresenta propriedades ecotoxicológicas. Na vida marinha, os peixes são os seres vivos mais afetados pelo descarte de metais pesados por afluentes industriais. Para esses animais, o cobre é o elemento mais tóxico depois do mercúrio, sendo que para os de água salgada esta toxicidade é amenizada devido à alta capacidade de complexação deste meio (NETO ALMEIDA, 2007).
A legislação Brasileira, através da resolução CONAMA (Conselho Nacional do meio Ambiente) $n^{\circ} 357$ de 17/03/2005, estabelece como limite máximo para emissão de cobre dissolvido em efluentes o valor de $1 \mathrm{mg} / \mathrm{L}$.

As principais técnicas de tratamento disponíveis para remoção de metais envolvem processos de precipitação, degradação química, fotoquímica e adsorção (OLIVEIRA, 1992). Dentro desses processos destaca-se a adsorção, por ser um método químico rápido e de baixo custo e com grande eficiência para remoção de metais pesados em baixa concentração.

Legaly (1994) estudou a capacidade de remoção de $\mathrm{Cu}^{2+}$ e $\mathrm{Mn}^{2+}$ em diferentes argilas e foi observado que a preferência da argila pelos íons $\mathrm{Cu}^{2+}$ diminuiu com o aumento da fração molar dos íons $\mathrm{Cu}^{2+}$ na solução.

A adsorção simultânea de $\mathrm{Cu}^{2+}, \mathrm{Cd}^{2+}$ e $\mathrm{Ni}^{2+}$ me soluções aquosas foi investigada por Sousa et al.(2001) utilizando alumina Al-55. Neste estudo, observou-se que $\circ \mathrm{Cu}^{2+}$ foi mais adsorvido que o $\mathrm{Cd}^{2+}$ e $\circ \mathrm{Ni}^{2+}$.

A argila em geral é um mineral natural, de granulação fina, terroso e que apresenta em sua estrutura silicatos hidratados formando tetraedros de silício e oxigênio e octaedros de alumínio, oxigênio e hidroxila. Dentro do grupo das sílicas a perlita se destaca por apresentar um baixo custo e um alto potencial de adsorção (ALMEIDA NETO, 2007). A perlita é um argilomineral de origem vulcânica vitrificada, um silicato de alumínio amorfo metaestável. O tamanho deste aluminossilicato é aumentado de 8 a 20 vezes quando é submetido a uma faixa de temperatura de 760 a $2000{ }^{\circ} \mathrm{C}$. O processo de expansão da perlita é comparado com a formação da pipoca. A água presente na estrutura molecular da perlita quando submetida ao rápido aquecimento é vaporizada formando bolhas, causando um inchaço e aumento do volume inicial (SOUZA, 2010). Quase toda a perlita é consumida na forma expandida, apesar da forma não expandida ter algumas aplicações. A Turquia possui a maior reserva de perlita, do mundo com cerca de $70 \%$ e produção total de $70 \times 10^{9}$ de toneladas ao ano. Ela pode ser encontrada em diferentes formas, tais como: granular, pó e esfoliante (ALKAN, 2005).

A perlita expandida pode ser aplicada em diversas áreas, como na construção civil, isolantes (térmico e acústico), horticultura, filtro e blocos refratários. A perlita pode ser também 
utilizada em tintas, vidro, plásticos, resinas e esmaltes. Esse tipo de argila é utilizado como catalisador em reação química, inibidor de oxidação e como abrasivo (ALKAN,1998; SOUZA, 2010).

$\mathrm{Na}$ área ambiental, diversos trabalhos têm sido publicados utilizando a perlita como agente adsorvente de inseticidas, pesticidas, matérias têxteis, resíduos radioativos e de metais pesados. A capacidade de adsorção desse mineral é aumentada após um tratamento com reagentes orgânicos tornando o hidrofóbico (BASTANI, 2006). As propriedades da perlita expandida fazem com que o mineral seja útil em muitas aplicações industriais inclusive na remoção de metais pesados nos afluentes como cádmio, cobre, chumbo, manganês, mercúrio e entre outros (SOUZA, 2010). A perlita expandida tem interessantes propriedades físico-químicas por ser termicamente inerte, ter alta porosidade, baixo peso molecular, não corrosivo, não absorve água, facilidade de encontrar e de ser um adsorvente ambientalmente correto.

A quitosana (Figura 1) é um biopolímero obtido a partir da desacetilização dos grupos amina da quitina, um dos mais abundantes polissacarídeos encontrados na natureza. A quitosana é um polieletrólito catiônico (em meio ácido) que possui diversos grupos reativos (hidroxila, acetamido, amino). Este polímero natural vem despertando diversas áreas devido as suas características e propriedades químicas favoráveis a aplicações diversas. Entre suas propriedades destacam-se atoxicidade, biocompatibilidade e biodegrabilidade. Os polímeros, em forma geral, possuem um caráter neutro ou ácido, já a quitosana é um polissacarídeo básico, mostrando uma característica particular em relação aos outros polissacarídeos (SIGNINI,1998).

$\mathrm{Na}$ cadeia molecular da quitosana, os átomos de nitrogênios estão na forma de amino alifáticos primários e, assim, sofrem reações peculiares de aminas. As cargas positivas da quitosana vem dos grupos amino desacetilizado, quando protonados em $\mathrm{pH}<6.0$. Para as poliaminas, quanto maior o número de grupos protonados maior será a dissociação do ácido conjugado (R-N $\left.\mathrm{NH}_{3}^{+}\right)$, devido à repulsão eletrostática entre eles (MELO, 2007).

A quitosana pode ser obtida por diversas formas, como gel, fibras ocas, esferas, cápsulas, membranas, entre outras, oferecendo vantagens nas aplicações comerciais, biomédica, indústrias e ambientais (KUMAR,2000). A quitosana é solúvel em ácidos orgânicos levemente fracos como por exemplo, os ácidos acético, fórmico e o lático. A solução de quitosana apresenta uma alta viscosidade devido ao seu alto peso molecular. Dependendo do grau de desacetilação pode variar de 50 e 1000 Da.

Nas últimas duas décadas, tem sido intensificada o número de publicações em diversas áreas (biomédica, farmacêutica, alimentícia, agricultura, tratamento de água) relacionadas á potencialidade da quitosana e a sua modificação química, devido ao seu baixo custo e propriedades químicas (SHAHIDI,1999). $\mathrm{Na}$ área ambiental, esse polímero vem sendo aplicado devido sua alta potencialidade de adsorção de metais pesados (cobre, cádmio, zinco e entre outros) em processos de purificação de água. A elevada capacidade adsortiva está relacionada ao grau de desacetilação e a massa molecular relativa (MELO,2007).

O desenvolvimento e aplicação de eletrodos modificados com quitosana tem apresentado crescente interesse nas áreas de ciência e tecnologia, devido a quitosana ser uma espécie quimicamente ativa e ter boas características físico-químicas (reatividade, seletividade, etc.) possibilitando assim uma resposta adequada para diversos propósitos e aplicações nas eletroanálises e sensores químicos (JANEGITZ, 2007).

Janegitz (2007) estudou a determinação de íons $\mathrm{Cu}(\mathrm{II})$ utilizando o eletrodo de pasta de carbono modificado com quitosana por voltametria de redissolução anódica, conseguindo um intervalo de concentração de cobre de $2,0 \times 10^{-7}$ a $7,4 \times 10^{-6} \mathrm{~mol} \mathrm{~L}^{-1}$.

Este trabalho tem como objetivo avaliar a potencialidade da perlita expandida na adsorção de íons cobre utilizando um eletrodo de carbono vítreo modificado com quitosana (EMQ).

\section{Material e Métodos}

A perlita expandida (fornecida pela Schumacher Insumos para Indústria) foi submetida à secagem numa estufa por $5 \mathrm{~h}$ a uma temperatura de $150{ }^{\circ} \mathrm{C}$. Posteriormente foi peneirada, para obtenção de uma fração granulométrica de 100 mesh.

A solução de quitosana foi preparada pela 
dissolução de $8,2 \mathrm{mg}$ em $5 \mathrm{~mL}$ de ácido acético (2\%) sob agitação por 2 horas.

Um eletrodo de carbono vítreo, com área geométrica de $2,0 \mathrm{~mm}^{2}$, foi usado para a construção do eletrodo modificado de quitosana (EMQ).

Para a modificação do eletrodo, foi adicionada uma alíquota de $25 \mu \mathrm{L}$ da solução de quitosana cobrindo a superfície do eletrodo vítreo. Em seguida foi seca a temperatura ambiente por 30 minutos. Posteriormente o EMQ foi mergulhado em $20 \mathrm{~mL}$ de ácido sulfúrico (3\%) para a obtenção do branco.

A solução contendo os íons cobre foi preparada a partir do $\mathrm{Cu}(\mathrm{SO})_{4} \cdot 5 \mathrm{H}_{2} \mathrm{O}$ em água ultra-pura, na concentração de $1 \times 10^{-2} \mathrm{molL}^{-1}$ de íons cobre. Esta solução foi posteriormente diluída para uma concentração de $500 \mathrm{mgL}^{-1}$ de $\mathrm{Cu}^{2+}$.

As análises foram realizadas em um potenciostato/galvanostato Autolab PGSTAT 12 (Eco Chemie) gerenciado pelo software GPES 4.9. A análise foi realizada numa célula eletroquímica convencional com capacidade para $50 \mathrm{~mL}$ de solução, utilizando-se um sistema de três eletrodos: eletrodo de trabalho de carbono vítreo (diâmetro $2,0 \mathrm{~mm}$ ), eletrodo de referência de $\mathrm{Ag} / \mathrm{AgCl}$ com KCl 3,0 $\mathrm{molL}^{-1}$ e um eletrodo de platina como auxiliar.

Foram realizados, inicialmente, estudos de otimização dos parâmetros operacionais da técnica de voltametria de pulso diferencial, como: Tempo de eletrodeposição $\left(t_{e d}\right)$, potencial inicial $\left(E_{i}\right)$, potencial final $\left(E_{f}\right)$, Potencial de degrau $\left(E_{d}\right)$, velocidade de varrredura(v) e amplitude de potencial de pulso aplicado( $\left(\mathrm{EE}_{\mathrm{p}}\right)$. As melhores condições obtidas para estes parâmetros foram: $t_{e d}=60 s, E_{i}=-0,3 V, E_{f}=0,5 V, E_{d}=-0,006 \mathrm{~V}, v=20$ $\mathrm{mVs}^{-1}$ e $D E_{p}=0,05 \mathrm{~V}$. A perlita foi utilizada como adsorvente de íons cobres. Foi pesada 0,50 e $1,00 \mathrm{~g}$ de perlita expandida e submetida à agitação magnética variando o tempo de 5 a 30 minutos, em intervalos de 5 minutos para avaliação. A concentração da solução de íons cobre foi de $500 \mathrm{mgL}^{-1}$. Após o tempo da adsorção, foram retiradas alíquotas, no volume de $3 \mathrm{~mL}$ e submetidas a centrifugação, com a finalidade de remover a perlita. Dessa alíquota, retira-se $1 \mathrm{~mL}$ que foi adicionado a célula eletroquímica juntamente com $20 \mathrm{~mL}$ de ácido sulfúrico $3 \%(\mathrm{v} / \mathrm{v})$. Em seguida, essa célula foi submetida às medidas voltamétricas com 0 eletrodo de trabalho de carbono vítreo modificado com quitosana, eletrodo de $\mathrm{Ag} / \mathrm{AgCl}$ como referência e como o auxiliar o eletrodo de platina (Figura 2). Todos os parâmetros foram avaliados a fim de se obter o melhor desempenho do sensor na determinação de íons $\mathrm{Cu}(\mathrm{II})$.

\section{Resultados e conclusões}

Foi avaliado o potencial ideal para o prétratamento de $\mathrm{Cu}^{2+}$ no EMQ com quitosana no potencial de $-0,3 \mathrm{~V}$ em um intervalo de 0 a $60 \mathrm{~s}$, adicionando diferentes alíquotas de solução de íns cobre $1,0 \times 10^{-2} \mathrm{molL}^{-1}$. O tempo de prétratamento que proporcionou um melhor perfil voltamétrico, foi o de $2 \mathrm{~s}$, sendo este tempo adotado para as medidas eletroquímicas.

O método utilizado para obtenção da curva de calibração foi à adição padrão. A curva analítica foi obtida com o eletrodo de carbono vítreo modificado com quitosana. Realizaram-se medidas voltamétricas de pulso diferencial no intervalo de potencial de $-0,3$ a $0,5 \mathrm{~V}$ (vs $\mathrm{Ag} / \mathrm{AgCl}$ ), velocidade de varredura de $20 \mathrm{mVs}^{-1}$, tempo de pulso de $60 \mathrm{~s}$ em $\mathrm{H}_{2} \mathrm{SO}_{4} 3 \%(\mathrm{v} / \mathrm{v})$ como eletrólito de suporte, intervalo de concentração de cobre de $7,94 \times 10^{-5}$ a $4,76 \times 10^{-4} \mathrm{moll}^{-1}$, a fim de se obter a curva analítica. A Figura 3 apresenta os voltamogramas de pulso diferencial obtidos nas condições experimentais descritas, mostrando pico anódico na região de $0,05 \mathrm{~V}$. $\mathrm{Na}$ Figura 4, é mostrado curva analítica, representada pela equação $y=6,22 \cdot 10^{-4} x-3,61$ $10^{-8}, R^{2}=0,963(n=19)$ para a concentração de $\mathrm{Cu}(\mathrm{II})$, com o limite de detecção de $1,7 \times 10^{-7}$ moll ${ }^{-1}$

O efeito da adsorção foi verificado em função da massa e do tempo de contato da perlita. No voltamograma de pulso diferencial do cobre em eletrodo modificado com quitosana os picos de oxidação apresentam intensidades de potenciais fixos $(\mathrm{Epc}=0.05 \mathrm{~V})$.

A Figura 5, representa o voltamograma após o tempo de contato de 5 a 30 min em 500 $\mathrm{mg} / \mathrm{L}$ de íons cobre com 1,00 g de perlita, no qual é observado dois picos na região anódica caracterizando a presença de íons cobre. Com aumento do tempo de contato da perlita com a solução deste íon metálico, diminui a área dos picos indicando a adsorção de íons cobre pela perlita.

Pelos resultados observou-se uma rápida remoção de cobre pela perlita expandida. Nos 
primeiros $5 \mathrm{~min}$ alcançou eficiência média de adsorção de $67,02 \%$. No tempo de $20 \mathrm{~min}$, a diferença entre a média de adsorção de $0,50 \mathrm{~g}$ e $1,00 \mathrm{~g}$ é de $2,79 \mathrm{mg} / \mathrm{L}$, observando um equilíbrio de adsorção do cobre até o tempo final de 30 $\min$.

No tempo de $30 \mathrm{~min}$ com $0,50 \mathrm{~g}$ de perlita foi observado o maior percentual de adsorção com $77,00 \%$ de eficiência com o desvio padrão de 2,83 entre os experimentos realizados com esta massa neste tempo de contato. Pode-se observar que para as duas massas de perlita a adsorção atingiu a eficiência máxima no tempo de 30 e 15 minutos para 0,50 e 1,00 g de perlita, respectivamente.

A perlita expandida mostrou ser um rápido adsorvente de íons cobre em solução. No tempo de $1 \mathrm{~h}$, a adsorção foi tão eficaz que não foi possível quantificar o percentual de adsorção através da análise eletroquímica. Para o estudo do efeito de massa da perlita, observou-se que

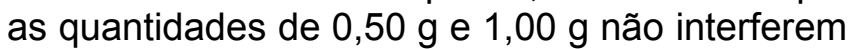
no processo de adsorção, na qual no tempo de 30 min o desvio padrão para a adsorção as diferentes massas foi de 9,14.

$\mathrm{Na}$ técnica eletroanalítica, o eletrodo de carbono vítreo modificado com quitosana pode ser empregado para a determinação de íons no intervalo de $7,94 \times 10^{-5}$ a $4,16 \times 10^{-4} \mathrm{~mol} \mathrm{~L}^{-1}$, empregando a voltametria de pulso diferencial. Esta espécie apresenta um comportamento voltamétrico de - 0,3 a 0,5 V (vs. $\mathrm{Ag} / \mathrm{AgCl}$ ), em um eletrólito de $\mathrm{H}_{2} \mathrm{SO}_{4}$.

Todas as técnicas utilizadas neste trabalho têm a característica de ser rápida, não destrutiva, apresentando alta sensibilidade e de baixo custo.

Considerando os resultados obtidos para a massa de 0,50 g de perlita, observou-se que a adsorção atingiu a eficiência máxima no tempo de 30 minutos, correspondendo a um percentual de $77,00 \%$ de cobre, enquanto para a massa de $1,00 \mathrm{~g}$, o máximo de adsorção ocorreu em 15 minutos com $75,41 \%$. No entanto, utilizando a média do percentual de adsorção para todos os tempos avaliados, foi verificado para 0,50 e 1,00 g a porcentagem de adsorção de $73,60 \%$ e $72,03 \%$, respectivamente. Esse fato demonstra que a massa da perlita não alterou significativamente o processo de adsorção.

\section{Agradecimentos}

Os autores agradecem à PETROBRAS pelo apoio financeiro.

\section{Referências}

1. ALKAN, Mahir. et al. Adsorption of CTAB onto perlite samples from aqueous solutions. Journal of Colloid and Interface Science, v. 291, p. 309-318, 2005.

2. ALKAN, Mahir. Surface titrations of perlite suspensions. Journal of Colloid and Interface Science. v. 207, p. 90-96, 1998.

3. ALMEIDA NETO, Ambrósio Florêncio. Desempenho da argilas bentoníticas na remoção de cátions de cádmio e cobre em colunas de leito fixo. Campina Grande (Tese de Mestrado, Universidade Federal de Campina Grande), 2007.

4. BASTANI, Dariush et al. Study of oil soption by expanded perlite at 298,15 k. Separation and Purification Technology, v. 52, p. 295-300, 2006.

5. CONAMA. Resolução $n^{\circ} 357$, de 17 de março de 2005.

6. FAQUIM, Elaine de Souza. Estudo da determinação de cobre por análise por diluição isotópica subestequiométrica. (Dissertação de Mestrado em ciências na área tecnologia nuclear, São Paulo), 1994.

7. HOMEM, Eduardo Machado. remoção de chumbo, niquel e zinco em zeólita utilizando sistemas de leito fluidizado. Dissertação de Mestrado, Faculdade de Engenharia Química, UNICAMP, 2001.

8. JANEGITZ, Bruno Campos; MARCOLINO JUNIOR, Luis Humberto; FATIBELLO FILHO, Orlando. Determinação voltamétrica por redissolução anódica de $\mathrm{Cu}(\mathrm{II})$ em águas residuárias empregando um eletrodo de pasta de carbono modificado com quitosana. Química Nova v. 30, N. 7, p 1673-1676, 2007.

9. KUMAR, Ravi. A review of chitin and chitosan applications. React.Funct. Polym., v.46, p. 1-27, 2000.

10. LAGALY, Gehard. Prog. Colloid Polym. Sci.,97,341, 1994. Em AGQUIAR, 
Mônica Regina Marques. Palermo de; NOVAES, Amanda Cardoso; GUARINO, Alcides Wagner Serpa. Remoção de metais pesados de efluentes industriais por aluminosilicatos. Química Nova,v.25, n 6B, p 1145-154, 2002.

11. MELO, Acácia Maria Santos. Estudo eletroquímico da trifluralina adsorção em quitosana, interação com o biossensor dsDNA e toxidade frente ao caramujo biomphalaria glabatra. Alagoas (Tese de Doutorado, Universidade Federal da Alagoas), 2007.

12. OLIVEIRA, Sérgio de Souza; JORDÃO, Cláudio Pereira; BRUNE Walter. Ambiente, 6,41, 1992.

13. SHAHIDI, Fereidoon; ARACHCHI, Janak Kamil Vidana; JEON, You-Jim. Food Applications of Chitin and Chitosans. Trends in Food Sci. Tecnhol., v. 10, p. 37-51, 1999.

14. SIGNINI, Roberto. Estudos de obtenção, purificação e caracterização de quitosana (Dissertação de Mestrado, Instituto de Química de São Carlos, Universidade de São Paulo), 1998.

15. SOUZA SANTOS, Persio. Ciência e Tecnologia de Argilas. $2^{\mathrm{a}}$ ed., São Paulo: Ed. Edgard Blücher Ltda., v. 1-3, 1992.
16. SOUZA, Hiale Yane Silva de. Hidrofobização da perlita para uso na adsorção de compostos orgânicos presentes nas águas produzidas de petróleo (Tese de Mestrado, Universidade Federal do Rio Grande do Norte-UFRN), 2010.

17. INSTITUTO ADOLFO LUTZ. Métodos físico-químicos para análise de alimentos. 4. ed. Brasília, 2005.

18. JANSON LT, kling $S$, Dallman PR. Anemia in children with acute infections seen in a primary care pediatric outpatient clinic. Pediatry Infect Dis 1986; 4:424-7.

19. Manual de fortificação de fubá e flocos de milho com ferro, Rio de Janeiro. Embrapa Agroindustria de Alimentos, novembro de 2001.

20. OMS. Série Informe Técnicos. Anemia nutricionales: informe de um grupo de expertos em nuticion de La OMS; 1972.

21. OMS. Série Informes Técnicos, 182. Anemia nutricionales: informe de um grupo de expertos em nutricion de La OMS> Ginebra: OMS; 1959.

22. VORA, A.; Thermal stability of folic and associated excipients, M.Sc. thesis, The University of Toledo, December 2001.

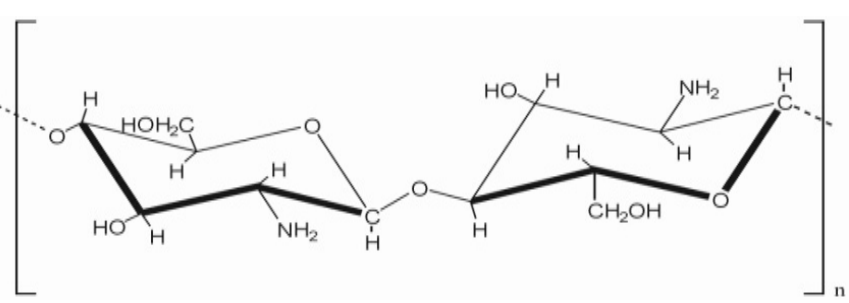

Figura 1: Estrutura química da quitosana

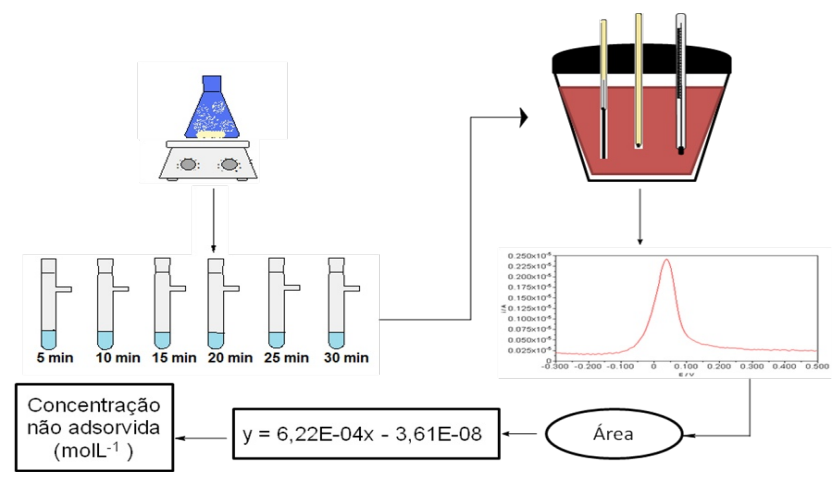

Figura 2 - Procedimento experimental realizado na remoção dos íons cobre e quantificação eletroanalítica. 


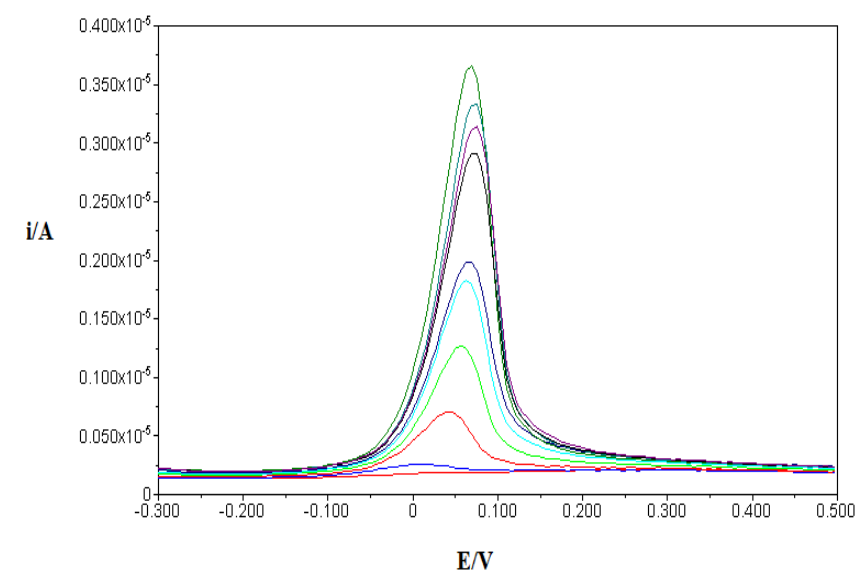

Figura 3 - Voltamogramas de pulso diferencial para íons $\mathrm{Cu}^{2+}$, obtidos mediante o método de adição de padrão.

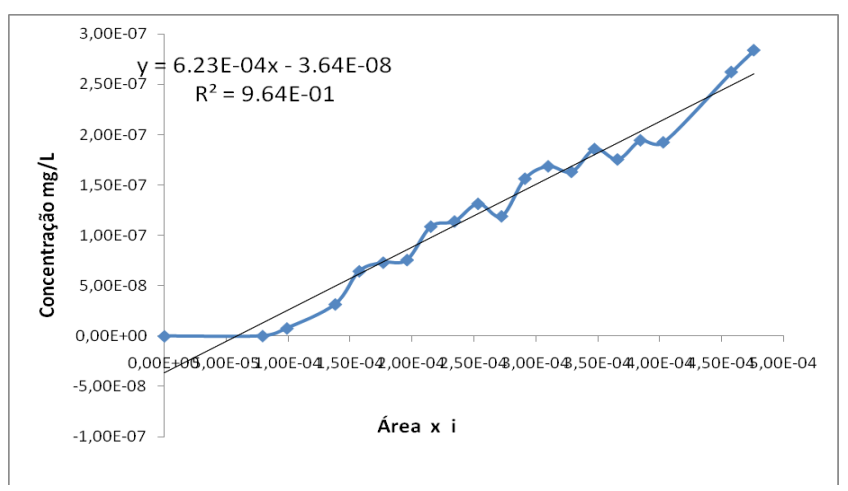

Figura 4 - Curva analítica da concentração de íons $\mathrm{Cu}^{2+}$ em função do sinal elétrico (ou área do pico) obtido na voltametria de pulso diferencial.

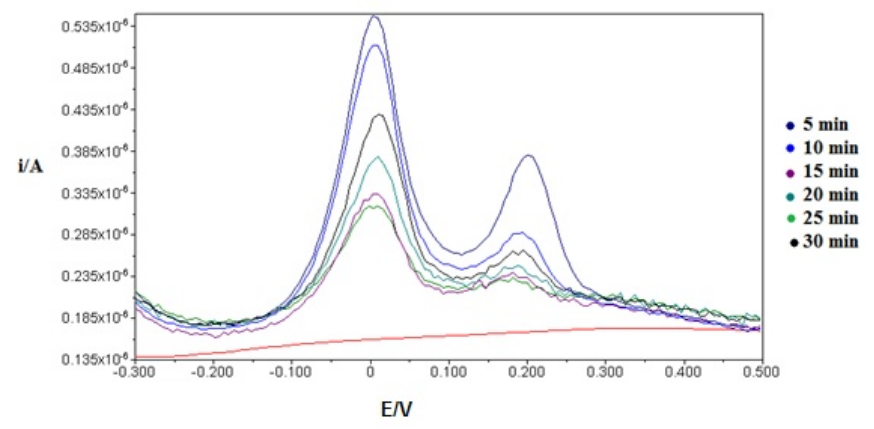

Figura 5 - Voltamogramas de pulso diferencial para íons $\mathrm{Cu}^{2+}$ em função do tempo, utilizando 1,00 g de perlita como adsorvente. 